\title{
Low-Cost Synthesis and Characterization of Silver Nanoparticles for Diverse Sensing Application
}

\author{
Sarah Akhtar \\ Federal Urdu University of Arts, \\ Science and Technology, \\ Karachi, Pakistan \\ sarah@fuuast.edu.pk
}

\author{
Zahid Farid \\ Department of Electrical Engineering, \\ University of Science and Technology, \\ Bannu, Pakistan \\ zahidfarid@ustb.edu.pk
}

\author{
Hasham Ahmed \\ Federal Urdu University of Arts, \\ Science and Technology, \\ Karachi, Pakistan \\ hasamsiddiqui@gmail.com
}

\author{
Shayan Ali Khan \\ Department of Electrical Engineering, \\ University of Engineering and Technology, Taxila, Pakistan \\ shayan.alikhan@yahoo.com
}

\author{
Zeeshan Najam Khan \\ Center for Advanced Electronics and Photovoltaic Engineering, \\ Islamabad, Pakistan \\ shaani75@hotmail.com
}

\begin{abstract}
Silver (Ag) nanoparticles (NPs) are synthesized and characterized by a low-cost chemical reduction method. Silver nanoparticles (Ag NPs) have pre-occupied the consideration of the scientific community due to their wide range of functions, utility and industrial applications, particularly in the fields of sensing technologies and medicine (particularly their efficiency against microbes, the ability of healing the wound and antiinflammatory properties). Ag NPs are synthesized by a low-cost fabrication method. X-ray diffraction (XRD), scanning electron microscopy (SEM), energy dispersive X-ray diffraction (EDX) and photometry techniques are used in this work to identify their nature and potentiality for diverse applications in sensing technologies.
\end{abstract}

Keywords-scanning electron microscopy (SEM); energy dispersive $X$-ray diffraction (EDX); sodium dodecyl sulphate (SDS)

\section{INTRODUCTION}

The study of nano-sized particles has gained a lot of attention in research and technology due to their growing applications in electronic industry and health sciences. Improvements in fundamental understanding of NPs may yield better methods of their manufacturing [1]. Metal nano-sized particles have been the subjects of research and exploration due to their distinctive electronic, optical, mechanical, magnetic and chemical properties. Due to their size and large specific surface area [2], metallic NPs can be used in many application areas such as catalysis, electronics, and photonics [3].

\section{LITERATURE REVIEW}

Diverse research techniques have been found for the synthesis of the metallic NPs, including reverse miscellaneous processes, salt diminution, ultrasonic irradiation, microwave dielectric heating reduction, radiolysis, thermal synthesis, electrochemical synthesis, etc [4]. Nano sized particles of Ag have displayed fascinating characteristics [4]. Presently, such NPs have regained importance in research due to the rise of bacterial resistance against antibiotics. The idea of using $\mathrm{Ag}$ NPs as an anti-bacterial agent is comparatively new [5]. Because of their high reactivity due to their large surface to volume ratio, NPs have taken over in exhibiting a critical behavior in inhibiting bacterial growth in a liquid and solid medium. Ag may be used to eliminate organisms on textile fabrics/yarn or they can be used for water purification [6]. Opposing to germicide effects of ionic Ag, the greater antimicrobial effect may be enhanced with the smaller size of particles [7]. Ag NPs have been optimized via various techniques such as microwave irradiation, thermal decomposition, an electrochemical method, laser ablation and chemical synthesis [7]. The easiest and the most cost effective is the bulk-solution synthetic technique for metal nano-sized particles is the chemical reduction of metal salts/crystals. The manufacturing of nanoscale metal silver particles, of distinct morphologies and sizes by chemical reduction of $\mathrm{Ag}$ salts has been reported [8]. This artificial technique follows the reduction of an ionic salt in a suitable medium in the presence of surfactant using various reducing agents. Produced Ag NPs exhibit intense colors due to the Plasmon resonance absorption [9]. The metal exterior is the same as plasma, which has free electrons in the conduction band and also has positively charged nuclei.

\section{EXPERIMENTAL DETAILS}

\section{A. Preparation of $\mathrm{Ag} \mathrm{NPS}$}

Two stabilizing agents, sodium dodecyl sulphate (SDS) and citric acid were used for the synthesis of $\mathrm{Ag} \mathrm{NPs}, \mathrm{AgNO}_{3}$ solution $(1.0 \mathrm{mg}$ per $50 \mathrm{ml})$ and $6 \%(\mathrm{w} / \mathrm{w})$ SDS were used as a metal salt predecessor and a stabilizing agent, respectively. hydrazine hydrate $\left(\mathrm{N}_{2} \mathrm{H}_{4}-\mathrm{H}_{2} \mathrm{O}\right)$ solution with a concentration of $2.0 \mathrm{mM}$ and citric acid solution $(1.0 \mathrm{mg}$ per $50 \mathrm{ml})$ were used as reducing agents. The transparent colorless solution was sufficiently heated and transformed to the characteristic pale yellow when hydrazine hydrate $\left(\mathrm{N}_{2} \mathrm{H}_{4}-\mathrm{H}_{2} \mathrm{O}\right)$ was used as the 
stabilizing agent. The occurrence of color indicated the formation of Ag NPs. The Ag NPs were then purified by centrifugation $\left(21000 \mathrm{rpm}\right.$ at $\left.10^{0} \mathrm{C}\right)$. To remove excess silver ions, the silver colloids were washed with ethanol and dried powder of the Ag NPs was obtained for further analysis and characterization.

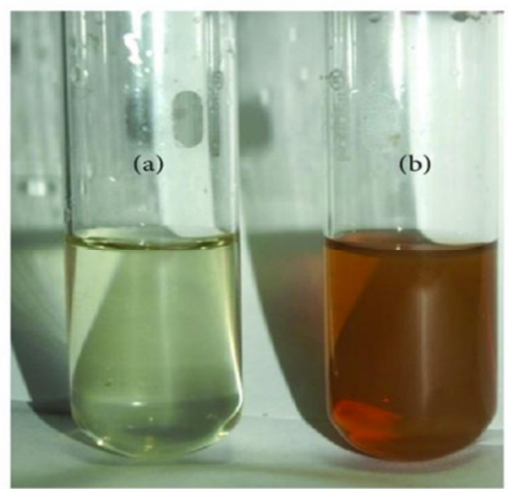

Fig. 1. a) $\mathrm{SDS}_{\text {and }}$ ) $\mathrm{AgNO}_{3}$ for the synthesis of Ag NPs

\section{B. X-Ray Diffraction (XRD) Technique}

Powder XRD data were collected on a Siemens D5000 powder diffraction meter using $\mathrm{Cu}$ radiation. Data were collected for angle $2 \theta$ ranging from $10^{\circ}$ to $90^{\circ}$, using a step size of 0.05 degree $/ \mathrm{sec}$ as placed in Table I. First, we grounded the sample to powdered form and place it in the center of the sample holder having dimensions of $5 \mathrm{~cm} \times 5 \mathrm{~cm}$. The sample was analyzed at random intervals. Phase and mineral identification of the sample were evaluated and analyzed by diffraction searching software comprising of over 136,895 patterns. This software contains an automated search-match option that compares the pattern of the sample by the International Center for Diffraction Data (ICDD) database.

TABLE I. XRD AND LATTICE SIZE OF AG NPS

\begin{tabular}{|c|c|c|c|c|c|c|}
\hline $\begin{array}{c}\text { Chord Mid. } \\
\mathbf{2 \theta}\end{array}$ & $\begin{array}{c}\mathbf{2 \theta} \\
(\mathbf{r a d})\end{array}$ & $\begin{array}{c}\text { FWHM } \\
\mathbf{2 \theta}\end{array}$ & $\begin{array}{c}\text { FWHM } \\
(\mathbf{r a d})\end{array}$ & $\begin{array}{c}\boldsymbol{D} \\
(\mathbf{n m})\end{array}$ & $\mathbf{h k l}$ & ${ }^{\mathbf{0}} \mathbf{A}$ \\
\hline 1.00 & 0.66 & 0.28 & 30.00 & 35.77 & 111 & 4.07 \\
\hline 44.38 & 0.77 & 0.38 & 0.00 & 29.29 & 220 & 5.77 \\
\hline 64.56 & 1.12 & 0.37 & 0.00 & 48.87 & 311 & 4.78 \\
\hline 77.54 & 1.35 & 0.42 & 0.00 & 86.18 & 511 & 6.39 \\
\hline \multicolumn{7}{|c|}{ Particle diameter $=50 \mathrm{~nm}$ and concentration $=1 \mathrm{mmol} / 50 \mathrm{ml}$}
\end{tabular}

\section{Sample Analysis}

XRD analysis (Figure 2) of the sample suggests the presence of rutile $\mathrm{Ag}$ in major phase according to ICDD No. 00-004-0783(1). The average crystallite size from the sharp peak is $50 \mathrm{~nm}$, which is estimated by using the Scherrer's formula.

\section{Microscopic Study}

SEM was undertaken by using the apparatus SEM, JSM6380 which provides information in relation to the size and morphology of the NPs. There was a fixed conductive carbon tape to an aluminum tip and the fine particles were placed on top of it. The spot sizes were 1,5 and $10 \mathrm{um}$ with a working distance of $2 \mathrm{~mm}$ and acceleration voltage set at $25.00 \mathrm{kV}$.

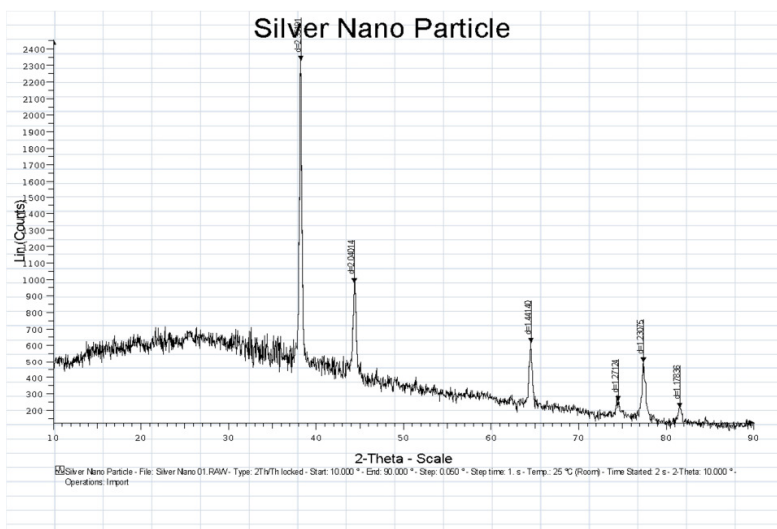

Fig. 2. X-Ray Diffraction (XRD) of Ag NPs with diameter 50nm.
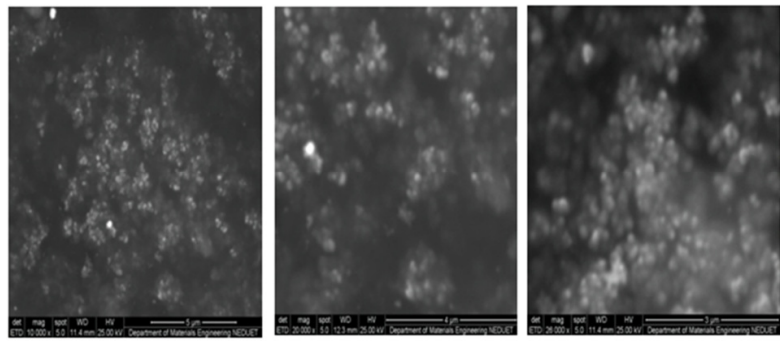

Fig. 3. SEM images of Ag NPs with diameter 50nm

TABLE II. EDX OF AG NPS BY USING ZAF METHOD

\begin{tabular}{|c|c|c|c|c|}
\hline Element & KeV & Mass\% & Error\% & At\% \\
\hline $\mathrm{C}$ & 0.277 & 11.72 & 0.53 & 18.69 \\
\hline $\mathrm{O}$ & 0.525 & 40.40 & 0.39 & 48.34 \\
\hline $\mathrm{Na}$ & 1.041 & 8.08 & 0.23 & 6.72 \\
\hline $\mathrm{Mg}$ & 1.253 & 1.90 & 0.21 & 1.49 \\
\hline $\mathrm{Al}$ & 1.486 & 1.10 & 0.19 & 0.78 \\
\hline $\mathrm{Si}$ & 1.739 & 31.36 & 0.18 & 21.37 \\
\hline $\mathrm{Ca}$ & 3.690 & 5.44 & 0.27 & 2.60 \\
\hline Total & 100.00 & \multicolumn{3}{|c|}{ Standard less quantitative analysis fitting coefficient: 0.3351} \\
\hline
\end{tabular}

Particle diameter $=50 \mathrm{~nm}$ and concentration $=1 \mathrm{mmol} / 50 \mathrm{ml}$

\section{E. Photometry (Ultraviolet Visible Spectroscopy (UV-VIS))}

We confirmed the presence of Ag NPs by measuring the wavelength of the sample in the spectrum UV-VIS of the PerkinElmer spectrophotometer LAMBDA 265 at a resolution of $2 \mathrm{~nm}(200 \mathrm{~nm}$ to $800 \mathrm{~nm})$. Plasmonic metal NPs have greater probability for biological and chemical sensor applications, because of their insightful spectral response to the local surroundings of the NPs facade and easy to monitor the light signal because of their sturdy scattering or absorption [10]. In the current study, we discovered the surface sensitivity dependence on Plasmon resonance (frequency and bandwidth) reaction to changes in their neighboring environment alongside the role of optical scattering on the shape and size of NPs and the kind of metal, which is, Ag. The conjectural concern with the surface Plasmon resonance situation exposed the spectral sensitivity. We can state that relative shift in resonance wavelength with regard to the refractive index change of neighboring materials, has two controlling factors: first is the bulk plasma wavelength, a property reliant on the metal nature, and second on the feature ratio of the element which is a 
geometrical factor [11]. It is shown that the sensitivity is directly proportional to both these parameters. In order to precisely discover the dependence of the spectral sensitivity on the discrete dipole, an estimate method was used for the calculation of optical spectrum of Ag [12]. Though, if we utilize smaller NPs they may stimulate an extreme expansion of the resonance spectrum [12]. This bounds the sensing resolution. The insensitivity of the Plasmon reaction for the metal composition is attributable to the reality that the bulk plasma frequency of the metal, which indicates the spectral dispersion of the factual dielectric function of metals and the surface Plasmon resonance state, has the same value for the pure metal [13]. The measured absorbance data can be correlated to the sample's concentration. Samples were synthesized by dispersion of the calculated quantity of sample (very diluted) and then $3 \mathrm{ml}$ of the solution was kept in a cuvette and submitted to the measurement.

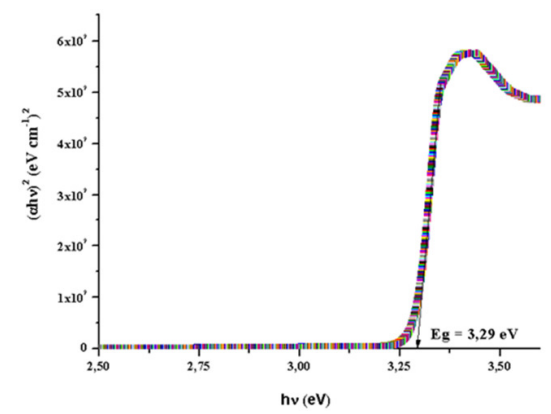

Fig. 4. Curve of $\mathrm{Ag}$ NPs a diameter of 50nm and mass concentration $1 \mathrm{mmol} / 50 \mathrm{ml}$

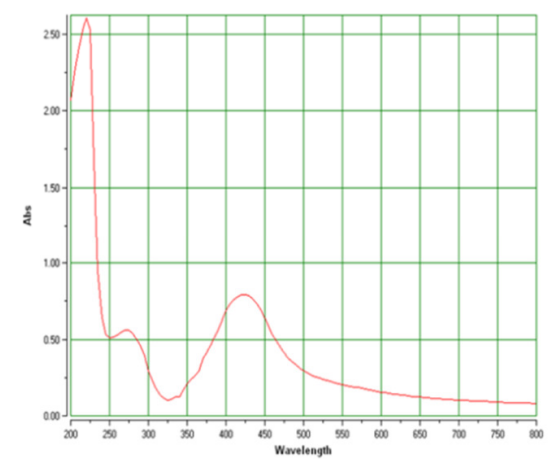

Fig. 5. Absorption spectra of silver nanoparticles with diameters ranging from $10 \mathrm{~nm}$ to $50 \mathrm{~nm}$ at mass concentrations of $1 \mathrm{mmol}$

\section{CONCLUSION}

Ag NPs were successfully synthesized using the simple and cost-effective chemical reduction method. XRD showed the formation of high purity Ag NPs. SEM and EDX viewgraphs confirmed the presence of NPs. From the photometry analysis we discovered the surface Plasmons and the resonance condition. It was apparent that the spectral sensitivity or relative shift in resonance wavelength with respect to the reflective index by varying neighboring materials has two controlling factors: the bulk plasma wavelength, a property reliant on the metal nature, and the aspect ratio of element which is a geometrical parameter. The optical band gap of Ag
NPs from the absorption spectrum by the Tauc's relation is calculated by [14]:

$$
E h v=B(h v-E g) n
$$

\section{ACKNOWLEDGMENT}

Authors are grateful to the Pakistan Council of Science and Industrial Research (PCSIR), Ministry of Science and Technology, Pakistan for their help in NP synthesis for this research. We are also thankful to the Dean of Sciences for providing the research fund.

\section{REFERENCES}

[1] A. L. Nogueira, R. A. F. Machado, A. Z. de Souza, F. Martinello, C. V. Franco, G. B. Dutra, "Synthesis and Characterization of Silver Nanoparticles Produced with a Bi-functional Stabilizing Agent", Industrial \& Engineering Chemistry Research, Vol. 53, No. 9, pp. 34263434,2014

[2] W. Zhang, X. Qiao, J. Chen, "Synthesis and characterization of silver nanoparticles in AOT micro-emulsion system", Chemical Physics, Vol. 330, pp. 495-500, 2006

[3] M. Rehan, H. M. Mashaly, S. Mowafi, A. A. El-Kheir, H. E. Emam, "Multi-functional textile design using in-situ Ag NPs incorporation into natural fabric matrix", Dyes and Pigments, Vol. 118, pp. 9-17, 2015

[4] P. F. Gutierrez, L. Olive, A. Banuelos, E. Orrantia, N. Nino, E. M. Sanchez, F. Ruiz, H. Bach, Y. Av-Gay, "Synthesis, characterization, and evaluation of the antimicrobial and cytotoxic effect of silver and titanium nanoparticles", Nanomedicine: Nanotechnology, Biology and Medicine, Vol. 6, No. 5, pp. 681-688, 2010

[5] P. Devaraj, P. Kumari, C. Aarti, A. Renganathan, "Synthesis and Characterization of Silver Nanoparticles Using Cannonball Leaves and Their Cytotoxic Activity against MCF-7 Cell Line", Journal of Nanotechnology, Vol. 2013, ArticleID 598328, 2013

[6] N. Singh, P. Khanna, "In situ synthesis of silver nano-particles in polymethyl methacrylate", Materials Chemistry and Physics, Vol. 104, No. 2, pp. 367-372, 2007

[7] S. Parveen, R. Misra, S. K. Sahoo, "Nanoparticles: a boon to drug delivery, therapeutics, diagnostics and imaging. Nanomedicine: Nanotechnology", Biology and Medicine, Vol .8, No. 2, pp. 147-166, 2012

[8] S. Sarkar, A. D. Jana, S. K. Samanta, G. Mostafa, "Facile synthesis of silver nano particles with the highly efficient anti-microbial property", Polyhedron, Vol. 26, No. 15, pp. 4419-4426, 2007

[9] N. Ghobadi, "Band gap determination using absorption spectrum fitting procedure", in: International Nano Letters, Vol. 3.No. 2, Springer, 2013

[10] R. Geethalakshmi, D. V. L. Sarada, "Characterization and antimicrobial activity of gold and silver nanoparticles synthesized using saponin isolated from Trianthema decandra L", Industrial Crops and Products, Vol. 6, No. 2, pp.107-115, 2013

[11] S. Baset, H. Akbari, H. Zeynali, M. Shafie, "Size measurement of metal and semiconductor nanoparticles via uv-vis absorption spectra", Digest Journal of Nanomaterials and Biostructures, Vol. 6, No. 2, pp. 709-716, 2011

[12] N. Sahu, D. Soni, B. Chandrashekhar, B. K. Sarangi, D. Satpute, R. A. Pandey, "Synthesis and characterization of silver nanoparticles using Cynodondactylon leaves and assessment of their antibacterial activity", Bioprocess and Biosystems Engineering, Vol. 36, No. 7, pp. 999-1004, 2013

[13] G. A. Valencia, L. C. D. O. Vercik, R. Ferrari, A. Vercik, "Synthesis and characterization of silver nanoparticles using water-soluble starch and its antibacterial activity on Staphylococcus aureus", Starch, Vol. 65, No. 11-12, pp. 931-937, 2013

[14] U. T. Khatoon, K. V. Rao, J. V. Rao, Y. Aparna, "Synthesis and characterization of silver nanoparticles by chemical reduction method", International Conference on Nanoscience, Engineering and Technology, Chennai, India, November 29-30, 2011 